\title{
Polarization-Selective Bidirectional Absorption Based on a Bilayer Plasmonic Metasurface
}

\author{
Tong Li ${ }^{1}$, Bin-Quan Chen ${ }^{1}$, Qian He ${ }^{1}$, Li-An Bian ${ }^{1}$, Xiong-Jun Shang ${ }^{1, *}$ and Guo-Feng Song ${ }^{2,3,4}$ \\ 1 Hunan Provincial Key Laboratory of Flexible Electronic Materials Genome Engineering, \\ Changsha University of Science and Technology, Changsha 410004, China; 1t@csust.edu.cn (T.L.); \\ BinquanChen2020@163.com (B.-Q.C.); hqian@csust.edu.cn (Q.H.); dk061bianlian@126.com (L.-A.B.) \\ 2 Institute of Semiconductors, Chinese Academy of Sciences, Beijing 100083, China; sgf@semi.ac.cn \\ 3 College of Materials Science and Opto-Electronic Technology, University of Chinese Academy of Sciences, \\ Beijing 100049, China \\ 4 Beijing Key Laboratory of Inorganic Stretchable and Flexible Information Technology, Beijing 100083, China \\ * Correspondence: xjshang.cn@gmail.com
}

Received: 26 October 2020; Accepted: 20 November 2020; Published: 23 November 2020

check for updates

\begin{abstract}
We propose an alignment-free and polarization-selective bidirectional absorber composed of a one-dimensional bilayer Au grating array buried in a silicon nitride spacer. The absorptivity of the designed structure is more than 95\% (77\%) under normal forward (backward) TM-polarized light incidence, and is more than $80 \%(70 \%)$ within a forward (backward) incident angle up to $30^{\circ}$. The great bidirectional absorption performance is illustrated by the resonance coupling of the surface plasmon polaritons (SPPs) resonance, the propagating surface plasmon (PSP) resonance and the localized surface plasmon (LSP) resonance under TM-polarized wave illumination. Moreover, the excitation of the Fano-like resonance mode of the proposed metasurface can produce two significantly different peaks in the absorption spectrum under the oblique TM-polarized incidence, which is beneficial for the plasmon-sensing application. Therefore, the proposed bidirectional metasurface absorber can be a candidate in the application of optical camouflage, thermal radiation, solar cells and optical sensing.
\end{abstract}

Keywords: polarization-selective; absorb; surface plasmons; metasurface

\section{Introduction}

Metasufaces, as extraordinary artificial structures, have attracted wide attention due to their outstanding nature in the context of many applications, such as electromagnetic wave stealth [1-3], imaging [4-6], flat metalens [7,8], optical filters [9], detectors and sensors [10,11]. Compared to the inherent bulk and loss of conventional optical materials, metasurfaces have unparalleled advantages in the integration and miniaturization of optical systems. However, metasurfaces still inevitably undergo some energy losses in their application. Optical absorbers can take the advantage of light loss to create a perfect absorption effect [12-18]. Perfect optical absorbers without the ability to reflect and transmit light have been the focus interested study for many years, due to their excellent absorption performance. Researchers concentrate their work on theoretical and experimental studies of optical absorbers with various structures, including gratings, nanoparticles, antenna arrays and multilayer composite structures. Many narrow-band, multiband and broadband metasurface-based absorbers have been investigated, and their operating bands covers from the visible region to the microwave band. They play an indispensable role in both science and practical applications, such as solar cells, thermal emitters, photovoltaic devices and sensing.

Polarization, as one of the most important properties of a light source, has attracted extensive attention from researchers. The polarization state can be detected by the flexible control of light 
absorption, transmission and reflection. Many metasurface-based absorbers (i.e., dielectric metasurfaces, plasmonic metasurfaces and composite metasurface) have been reported, and some promising applications have been discussed [19-23]. For example, metallic film with nanotrenches can strongly couple with polarized light to induce complete absorption, which applies to applications from ultrafast metallic photocathodes and the generation of laser harmonics, through field concentration, to polarization control and switching [24]. A high polarization sensitivity absorber can be used to enhance the contrast detection technology of the target, making up for the lack of intensity detection under complex camouflage conditions [19]. The latest report presents an active tunable polarization-sensitive multiband absorber based on graphene, which has potential applications in mid-infrared polarization-sensitive filters and detectors [25]. However, the designed polarization-selective structure can only absorb light waves from one direction, while reflecting light waves from another direction, which limits the practical application of the device. It is foreseeable that the realization of the bidirectional polarization-selective absorption of light waves can further expand the application of metasurface-based complete absorbers in optical detection.

In this paper, we theoretically demonstrate an alignment-free and polarization-selective bidirectional absorber composed of bilayer gratings buried in a silicon nitride spacer. The simulation results indicate that the absorptivity of the designed device is over $95 \%(77 \%)$, around $666 \mathrm{~nm}$ for normal forward (backward) TM-polarized incident light, and can be kept above $80 \%$ (70\%) within a forward (backward) incident angle up to $30^{\circ}$. The great bidirectional absorption performance is caused by the resonance coupling of surface plasmon polaritons (SPPs) resonance, propagating surface plasmon (PSP) resonance and localized surface plasmon (LSP) resonance under the excitation of TM-polarized incident light. Moreover, the Fano-like resonance mode of the designed structure can be excited under the illumination of TM-polarized oblique incident light, which is beneficial for sensing applications. Thus, it is expected that the proposed device has great potential in optical camouflage, thermal radiation, solar cells and optical sensing.

\section{Structure and Results}

Figure 1a illustrates the rendering of the proposed polarization-selective bidirectional perfect absorber, which consists of a one-dimensional array of bilayer gold grating buried in a silicon nitride spacer. The $x z$ plane schematic indicates the geometrical parameters of the double unit cells of the designed bilayer plasmonic grating structure, as shown in Figure $1 \mathrm{~b}$. The period of the one-dimensional array is $\mathrm{P}=320 \mathrm{~nm}$. The width of the narrow grating is $\mathrm{w}_{1}=45 \mathrm{~nm}$, and the gap between adjacent gratings is $g_{1}=275 \mathrm{~nm}$. The width of the wide grating is $\mathrm{w}_{2}=220 \mathrm{~nm}$, and the gap between neighboring gratings is $\mathrm{g}_{2}=100 \mathrm{~nm}$. The thickness of both gold gratings is $t=20 \mathrm{~nm}$. The thickness of the silicon nitride spacer between the bilayer gold gratings is $t_{1}=100 \mathrm{~nm}$ and the thickness of the silicon nitride spacer covered onto the gold grating is $t_{2}=80 \mathrm{~nm}$. Incident plane wave propagates in the $-z$ - (forward) or $+z$ - (backward) direction. The TM (or TE) incident wave represents the incident light with the electric field direction perpendicular (or parallel) to the grating, as shown in Figure 1b. The spectral response and field distribution of the structure are analyzed via finite element method (FEM) simulations using the commercial software COMSOL Multiphysics (Version 5.6, COMSOL Co., Ltd. 2D lujiazui financial services plaza, 1217 dongfang road, pudong new area, Shanghai, China). Periodic boundary conditions are used in the $x$-direction of a unit cell, and waveguide ports boundary conditions and perfectly matched layers are applied in the $z$-direction. The refractive index of silicon nitride is set to 2 . The optical constant of gold is taken from P. B. Johnson and R. W. Christy's work [26]. 
(a)

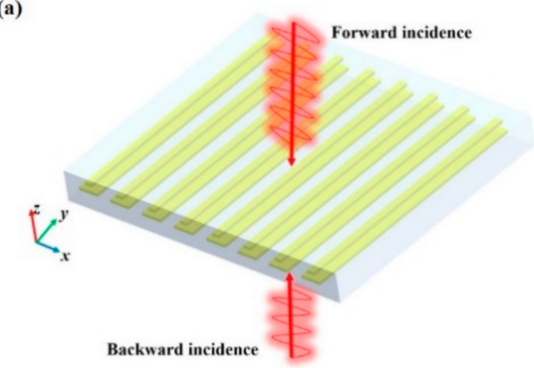

(b)

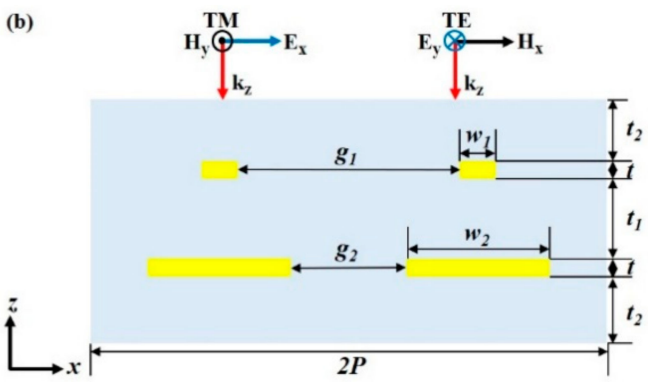

Figure 1. (a) Schematic illustration of the polarization-selective bidirectional absorber. (b) The double unit cells profile of the structure in the $x z$-plane.

The absorption, transmission and reflection spectra of the designed bilayer grating structure under forward TM-polarized incidence are indicated in Figure 2a. The reflection curve is near zero and an obvious decline can be observed in the transmission curve, while an absorption peak with over $95 \%$ absorptance is obtained around $666 \mathrm{~nm}$. Moreover, the near-perfect absorption of incident waves in the bilayer plasmonic metasurface is polarization-selective. Figure $2 \mathrm{~b}$ presents the absorption spectra of the proposed metasurface under both TM- and TE-polarized incidence. It can be clearly found that there is an absorption peak under TM-polarized irradiation, while the absorptance under TE-polarized irradiation is near zero around $666 \mathrm{~nm}$. Similarly, we calculate the absorption, transmission and reflection spectra of the designed bilayer grating structure under backward TM-polarized incidence, as shown in Figure 2c. The obvious drop is clearly visible in the transmission, and an absorption peak with over $77 \%$ absorptance appears around $666 \mathrm{~nm}$. Compared to forward incidence, the incident light first illuminates on the wide metal grating array with backward incidence, which creates a stronger reflection effect $(>21 \%)$ and weakens the absorption effect of the structure. Figure $2 \mathrm{~d}$ shows the absorption spectra under both backward TM- and TE-polarized incidence. The absorption peak only exists under TM-polarized illumination, and the absorptance is near zero under TE-polarized illumination. This means that the designed structure has the function of polarization-selective bidirectional absorption.
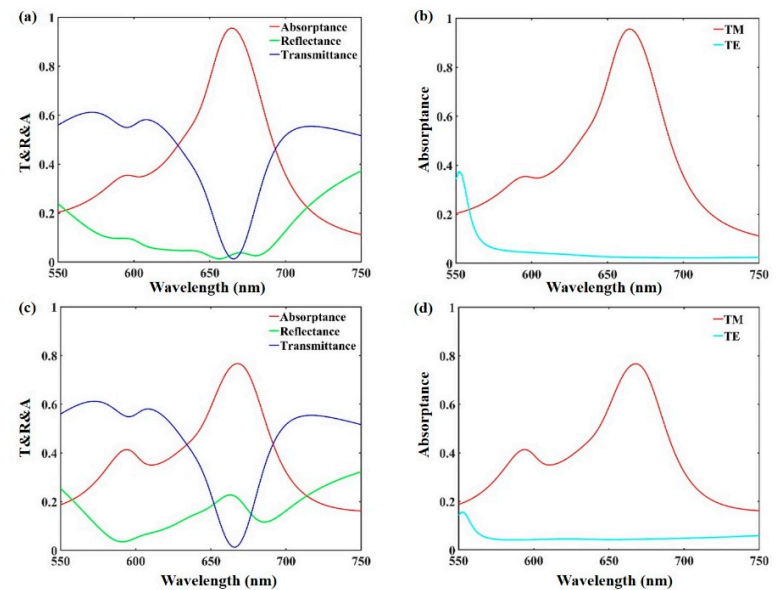

Figure 2. (a,c) show the absorption, transmission, and reflection spectra of the designed bilayer grating structure under forward and backward TM-polarized incidence, respectively. (b,d) show the absorption spectra of the proposed bilayer metasurface under forward and backward incident light with TE-polarized and TM-polarized irradiation, respectively.

\section{Analyses}

To reveal the physical mechanism of the metasurface-based polarization-selective bidirectional absorber, the current density $(J)$, and the electric and magnetic field distributions $(|E|$ and $|\mathrm{H}|)$, of the structure under TM-polarized incidence are simulated in the $x z$ plane. Figure 3 shows the field 
distribution of the designed structure at the resonance wavelength of $664 \mathrm{~nm}(668 \mathrm{~nm})$ under normal forward (backward) incidence. As can be seen from Figure 3a, the electric current is mainly distributed in the bilayer metal gratings under the incidence of forward TM-polarized light at a wavelength of $664 \mathrm{~nm}$. This means that the optical absorption loss is mainly caused by the metal grating. The electric field distribution in Figure $3 \mathrm{~b}$ indicates that the bilayer metal gratings induce the surface plasmon polaritons (SPPs) under the excitation of incident light. The electric field localizes around the metal corners and they couple to each other in the top-layer grating due to the narrow width, which results in a strong local surface plasmon (LSP) resonance. Due to the close distance between the two gratings, light is coupled into the $\mathrm{Si}_{3} \mathrm{~N}_{4}$ spacer between the bilayer gratings, and a weak coupling occurs with the propagating surface plasmon (PSP) resonance [27]. This can also be proven in the magnetic field distribution, as shown in Figure 3c. The magnetic field distribution is not only concentrated in the dielectric space layer above and below the metal grating, but is also significantly enhanced in the gap of the adjacent wide metal grating.
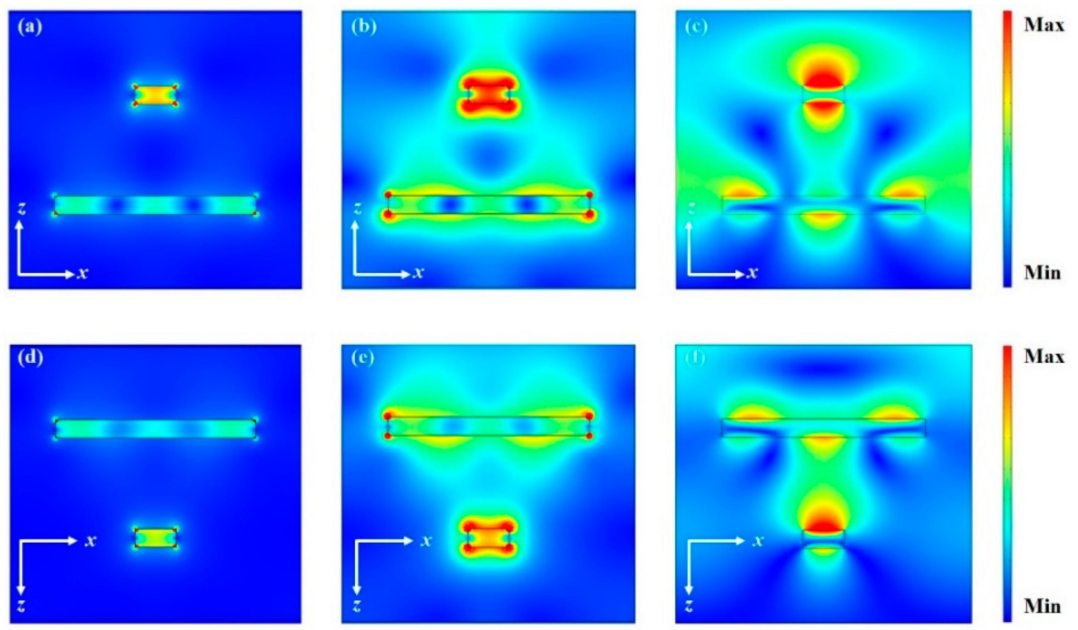

Figure 3. (a-c) Current density J, and electric and magnetic field distributions $|\mathrm{E}|$ and $|\mathrm{H}|$, respectively, of the structure under forward TM-polarized light incidence. (d-f) Current density J, and electric and magnetic field distributions $|\mathrm{E}|$ and $|\mathrm{H}|$, respectively, of the structure under backward TM-polarized light incidence.

To further verify the benefits of introducing the PSP resonance, we compare the absorption spectra of the proposed bilayer gold gratings with the narrow and wide single-layer gold gratings under the forward TM-polarized incidence, respectively. The layer thickness of the single-layer grating is identical to the proposed one. The thickness of the silicon nitride spacer covered onto the single-layer gold grating is $140 \mathrm{~nm}$. The absorption spectra of both cases are shown in Figure 4. We can see that the absorptivity of the bilayer grating is significantly stronger than that of the single-layer grating in the analyzed wavelength range. This is because the PSP resonance mode can be induced in the bilayer grating. The inset shows the electric field distribution $\left(\left|\mathrm{E}_{z}\right|\right)$ of two single-layer gratings with different widths at the resonance wavelength. The blue and green boxes represent the electric field distribution of the narrow grating and the wide grating, respectively. We find that a single-layer narrow grating produces fundamental mode resonance, while wide gratings produce high-order mode resonance around $666 \mathrm{~nm}$. The superposition of these two modes induces PSP mode resonance in the bilayer grating. This result also shows that the PSP resonance, SPP resonance and LSP resonance modes together lead to strong absorption at around $666 \mathrm{~nm}$. 


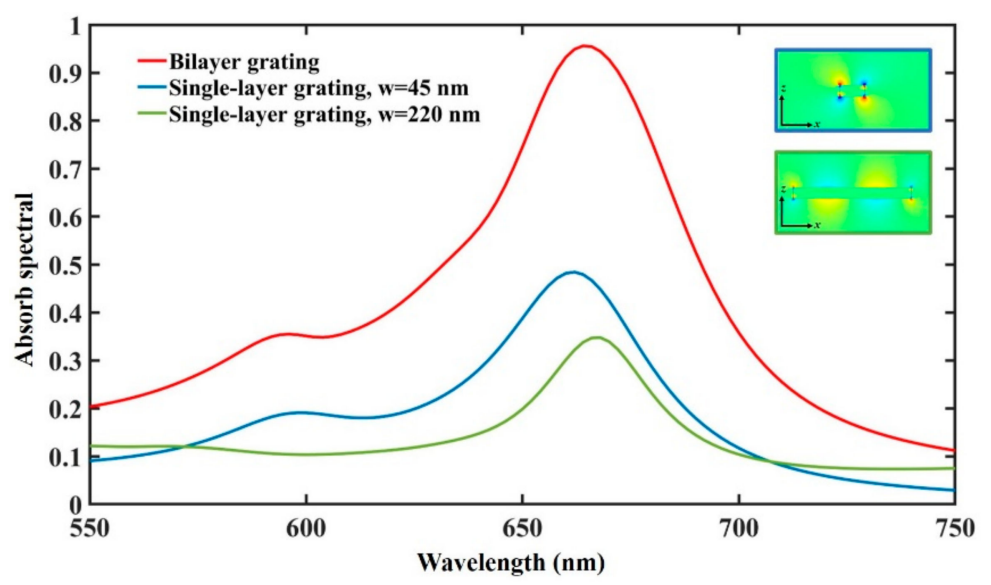

Figure 4. The absorption spectra of the proposed bilayer gold gratings (red line) with the narrow single-layer grating (blue line) and wide single-layer grating (green line), respectively. The blue and green boxes in the illustration represent the electric field distribution $\left(\left|\mathrm{E}_{\mathrm{z}}\right|\right)$ of the narrow grating and the wide grating at the resonance wavelength, respectively.

Compared to the forward incidence, the TM-polarized light excites the SPPs resonance of the wide grating under the backward incidence at a wavelength of $668 \mathrm{~nm}$. Similarly, the electric current is concentrated in the metal grating, as shown in Figure 3d. Figure 3e presents that the wide grating is strongly excited by the incident wave, and there are high electric field distributions at both ends of the grating. The resulting high-order mode resonance induces the LSP resonance of the narrow grating, which is placed $100 \mathrm{~nm}$ away from the wide grating. The high-order mode resonance generated by wide grating array reflects part of the incident light back into the air, which results in the absorptance of the bidirectional absorber under the backward TM-polarized wave incidence being lower than that of the forward incidence. In this case, LSP resonance dominates the absorption. The magnetic field is strongly confined in the $\mathrm{Si}_{3} \mathrm{~N}_{4}$ spacer between the bilayer gratings, as illustrated in Figure $3 \mathrm{f}$. The results illustrate that the narrow grating plays an important role in the light absorption, and the resonance coupling of SPPs resonance, PSP resonance and LSP resonance produced by the bilayer grating array under the excitation of forward (or backward) TM-polarized incident light leads to strong absorption. However, the resonance coupling effect of the proposed structure cannot be acquired under the TE-polarized wave excitation (not shown here), and the bidirectional absorption cannot be obtained. Therefore, the designed double-layer grating array structure has the characteristic of polarization-selective bidirectional absorption.

\section{Discussion}

The previous bilayer metasurface structures have relatively high requirements for alignment accuracy, which poses a great challenge to the fabrication and cost. Here, we discuss the influence of the alignment accuracy of the bilayer gratings on the absorption effect of the designed bidirectional absorber. Figure 5a presents the $x z$ plane of the device, where the narrow grating and the wide grating are not aligned, and the center misalignment distance in the $x$-direction between bilayer gratings is defined as $\mathrm{D}$. The simulation results of the absorption spectra for the structure with a different center misalignment distance D under normal forward and backward TM-polarized incidence are shown in Figure 5b,c, respectively. Figure $5 \mathrm{~b}$ shows that the absorptance keeps over $90 \%$ when the misalignment distance $\mathrm{D}$ is no more than $60 \mathrm{~nm}$, and the absorptance keeps over $80 \%$ when the misalignment distance D is no greater than $96 \mathrm{~nm}$. The absorptance even exceeds $75 \%$ when the misalignment distance D reaches $130 \mathrm{~nm}$, where the center position of the narrow grating is far from the wide grating in the $x$-direction. Similarly, Figure $5 \mathrm{c}$ shows that the absorptance can reach more than $80 \%$ when the misalignment distance D exceeds $26 \mathrm{~nm}$ under the backward TM-polarized incidence. Due to the displacement increase, the magnetic field is no longer limited to the dielectric space between the bilayer gratings, 
and significantly increases in the gap between adjacent narrow gratings, which induces PSP resonance and enhances the optical loss. These results indicate that the designed double-layer grating structure can realize bidirectional absorption without high alignment accuracy, which greatly reduces the sample fabrication requirements.
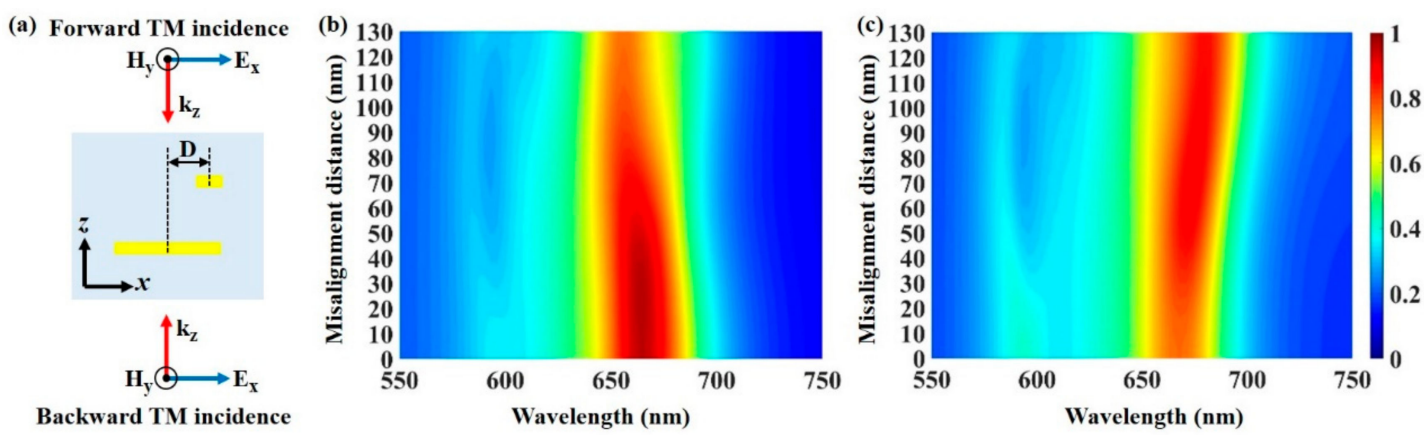

Figure 5. (a) Schematic of the center misalignment between the bilayer gratings. D indicates the center misalignment distance in the $x$-direction. $(\mathbf{b}, \mathbf{c})$ show the absorption spectra with different center misalignment distances, D, under forward and backward TM-polarized light incidence, respectively.

In addition, we investigate the influence of oblique incidence on the bidirectional absorption performance. Figure 6a indicates the simulation results of the absorption spectrum under forward TM-polarized incidence with different incident angles. It is obvious that a high absorption capability can be maintained at around $666 \mathrm{~nm}$ with the incident angle varying from 0 to $30^{\circ}$, which can be kept above $80 \%$. A similar absorptivity can also be seen in the absorption spectrum under backward TM-polarized incidence, as shown in Figure $6 \mathrm{~b}$. The absorption is more than $70 \%$ within an incident angle up to $30^{\circ}$. Thus, the designed polarization-selective bidirectional absorber device can retain a great performance at a wide range of incident angles. It is worth noting that an absorption dip appears when the incident angle increases. The asymmetric absorption spectrum can be obtained when the incident angle is more than $9^{\circ}\left(18^{\circ}\right)$ under forward (backward) TM-polarized incidence, which shows an obvious characteristic of Fano-like resonance (an absorption rise close to an absorption peak). In order to clearly show the Fano-like resonance spectral, we plot the absorption spectral under the forward and backward oblique TM-polarized light at the incident angle of $25^{\circ}$, as shown in Figure $6 \mathrm{c}$,d. According to the previous publications, the Fano-like resonance is induced by the interference and spatial overlap between continuous radiative resonance (bright mode) and nonradiative resonance (narrow dark mode) [28]. The Fano-like resonance in the designed structure is caused by the interference between the wide continuous spectrum resonance mode and the narrow dark resonance mode. The wide continuum resonance mode is the SPPs resonance mode of different orders on the metal grating surface, and the narrow dark resonance mode is caused by the asymmetry of the charge distribution in the metal slit. The light diffraction order is increased with the increase of the incident angle, and the symmetrical distribution of the enhanced electromagnetic field is gradually broken [29]. The left absorption peak corresponds to a continuum SPPs resonance mode with different orders on the bilayer grating, and the right narrow absorption peak is induced by the asymmetric charge distribution in the metal grating slit [30]. Therefore, the presented bilayer grating structure can maintain the function of polarization-selective bidirectional absorption within an incident angle up to $30^{\circ}$. The excited Fano-like resonance mode of the absorber at oblique incidence is conducive to the optical sensing application [29-34]. 
(a)

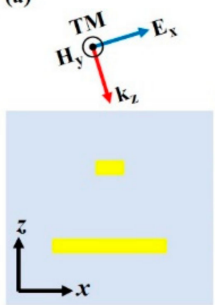

(c)
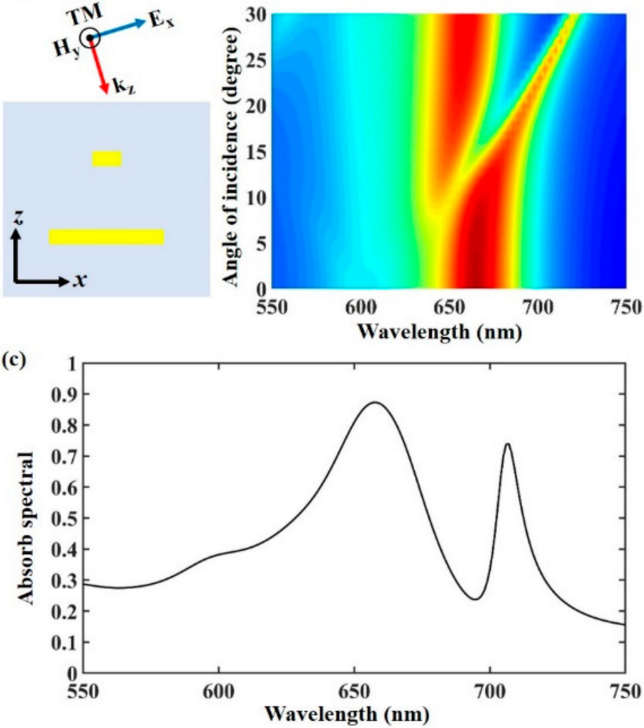

(b)

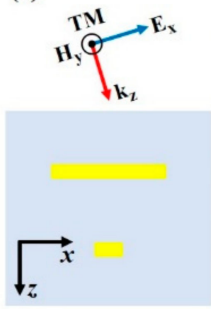

(d)

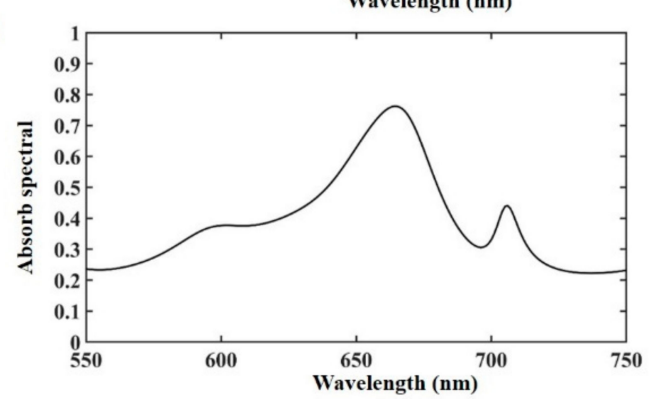

Figure 6. Absorption spectra of the proposed bilayer metasurface under forward (a) and backward (b) TM-polarized light incidence with different incident angles. The absorption spectral under forward (c) and backward (d) oblique TM-polarized light at the incident angle of $25^{\circ}$.

\section{Conclusions}

In conclusion, we have proposed a polarization-selective bidirectional absorber based on a bilayer metal grating array buried in a silicon nitride spacer. The simulation results indicate that the designed structure can realize polarization-selective absorption of TM-polarized optical waves around $666 \mathrm{~nm}$. The absorption is more than 95\% (77\%) under forward (backward) light incidence, under normal illumination. The SPPs resonance combines with PSP resonance and LSP resonance between bilayer metal gratings, causing this great absorption effect. The calculated results also show that the misalignment distance between bilayer gratings has little effect on the absorption efficiency of the metasurface-based absorber, which significantly reduces the fabrication requirement. The absorptivity remains higher than $80 \%(70 \%)$ for forward (backward) TM-polarized light within an incident angle up to $30^{\circ}$. Moreover, the Fano-like resonance mode of the proposed metasurface can be excited with the incident angle's increase, which is beneficial for the plasmon sensing application. Our design opens a door for the realization of polarization-selective bidirectional absorbers, which can be widely used in optical camouflage, thermal radiation solar cells and optical sensors.

Author Contributions: Conceptualization, T.L.; formal analysis, T.L.; visualization, T.L.; writing, original draft preparation, T.L.; writing, review and editing, T.L., B.-Q.C., Q.H., L.-A.B. and G.-F.S.; supervision, X.-J.S. All of the authors discussed the results and commented on the manuscript. All authors have read and agreed to the published version of the manuscript.

Funding: The Strategic Priority Research Program of Chinese Academy of Sciences (Grant No. XDB43010000). Education Department of Hunan Province (19C0035), Hunan University Student Innovation Project (202010536051), Natural Science Foundation of Hunan Province (2020JJ5565), Open Research Fund of Hunan Provincial Key Laboratory of Flexible Electronic Materials Genome Engineering (Grant No. 201909).

Conflicts of Interest: The authors declare no conflict of interest.

\section{References}

1. Pendry, J.; Schurig, D.; Smith, D. Controlling Electromagnetic Fields. Science 2006, 312, 1780-1782. [CrossRef]

2. Smith, D.; Schuring, D.; Mock, J.; Justice, B.; Cummer, S.; Pendy, J.; Starr, A. Metamaterial Electromagnetic Cloak at Microwave Frequencies. Science 2006, 314, 977-980. [CrossRef]

3. Ni, X.; Wong, Z.; Mrejen, M.; Wang, Y.; Zhang, X. An ultrathin invisibility skin cloak for visible light. Science 2015, 349, 1310-1314. [CrossRef] [PubMed] 
4. Ye, W.; Zeuner, F.; Li, X.; Reineke, B.; He, S.; Qiu, C.; Liu, J.; Wang, Y.; Zhang, S.; Zentgraf, T. Spin and wavelength multiplexed nonlinear metasurface holography. Nat. Commun. 2016, 7, 11930. [CrossRef] [PubMed]

5. Walter, F.; Li, G.; Meier, C.; Zhang, S.; Zentgraf, T. Ultrathin Nonlinear Metasurface for Optical Image Encoding. Nano Lett. 2017, 17, 3171-3175. [CrossRef]

6. Novotny, L.; Beversluis, M.; Youngworth, K.; Brown, T. Longitudinal Field Modes Probed by Single Molecules. Phys. Rev. Lett. 2001, 86, 5251-5254. [CrossRef]

7. Pendry, J. Negative Refraction Makes a Perfect Lens. Phys. Rev. Lett. 2000, 85, 3966-3969. [CrossRef]

8. Fang, N.; Lee, H.; Sun, C.; Zhang, X. Sub-Diffraction-Limited Optical Imaging with a Silver Superlens. Science 2005, 308, 534-537. [CrossRef]

9. Shah, Y.; Grant, J.; Hao, D.; Kenney, M.; Pusino, V.; Cumming, D. Ultra-narrow Line Width Polarization-Insensitive Filter Using a Symmetry-Breaking Selective Plasmonic Metasurface. ACS Photonics 2018, 5, 663-669. [CrossRef]

10. Pelzman, C.; Cho, S. Polarization-selective optical transmission through a plasmonic metasurface. Appl. Phys. Lett. 2015, 106, 251101. [CrossRef]

11. Panchenko, E.; Cadusch, J.; James, T.; Roberts, A. Plasmonic Metasurface-Enabled Differential Photodetectors for Broadband Optical Polarization Characterization. ACS Photonics 2016, 3, 1833-1839. [CrossRef]

12. Wu, D.; Liu, C.; Liu, Y.; Xu, Z.; Yu, Z.; Yu, L.; Chen, L.; Ma, R.; Zhang, J.; Ye, H. Numerical study of a wide-angle polarization-independent ultra-broadband efficient selective metamaterial absorber for near-ideal solar thermal energy conversion. RSC Adv. 2018, 8, 21054-21064. [CrossRef]

13. Wu, D.; Li, R.; Liu, Y.; Yu, Z.; Chen, L.; Ma, R.; Ye, H. Ultra-narrow Band Perfect Absorber and Its Application as Plasmonic Sensor in the Visible Region. Nanoscale Res. Lett. 2017, 12, 427. [CrossRef] [PubMed]

14. Zou, C.; Ren, G.; Hossain, M.; Nirantar, S.; Withayachumnankul, W.; Ahmed, T.; Bhaskaran, M.; Sriram, S.; Gu, M.; Fumeaux, C. Metal-Loaded Dielectric Resonator Metasurfaces for Radiative Cooling. Adv. Opt. Mater. 2017, 5, 1700460. [CrossRef]

15. Feng, A.; Yu, Z.; Sun, X. Ultranarrow-band metagrating absorbers for sensing and modulation. Opt. Express 2018, 26, 28197-28205. [CrossRef]

16. Takatori, K.; Okamoto, T.; Ishibashi, K. Surface-plasmon-induced ultra-broadband light absorber operating in the visible to infrared range. Opt. Express 2018, 26, 1342-1350. [CrossRef]

17. Qin, M.; Xia, S.; Zhai, X.; Huang, Y.; Wang, L.; Liao, L. Surface enhanced perfect absorption in metamaterials with periodic dielectric nanostrips on silver film. Opt. Express 2018, 26, 30873-30881. [CrossRef]

18. Gong, Y.; Wang, Z.; Li, K.; Uggalla, L.; Huang, J.; Copner, N.; Zhou, Y.; Qiao, D.; Zhu, J. Highly efficient and broadband mid-infrared metamaterial thermal emitter for optical gas sensing. Opt. Lett. 2017, 42, 4537-4540. [CrossRef]

19. Lee, D.; Han, S.; Jeong, Y.; Nguyen, D.; Yoon, G.; Mun, J.; Chae, J.; Lee, J.; Ok, J.; Jung, G.; et al. Polarization-sensitive tunable absorber in visible and near-infrared regimes. Sci. Rep. 2018, 8, 12393. [CrossRef]

20. Tang, J.; Xiao, Z.; Xu, K.; Ma, X.; Wang, Z. Polarization-Controlled Metamaterial Absorber with Extremely Bandwidth and Wide Incidence Angle. Plasmonics 2016, 11, 1393-1399. [CrossRef]

21. Meng, L.; Zhao, D.; Li, Q.; Qiu, M. Polarization-sensitive perfect absorbers at near-infrared wavelengths. Opt. Express 2013, 21, A111-A122. [CrossRef] [PubMed]

22. Pan, M.; Li, Q.; Hong, Y.; Cai, L.; Li, J.; Qiu, M. Circular-polarization-sensitive absorption in refractory metamaterials composed of molybdenum zigzag arrays. Opt. Express 2018, 26, 17772-17780. [CrossRef] [PubMed]

23. Tuan, T.; Hoa, N. Defect induced co-polarization broadband metamaterial absorber. AIP Adv. 2019, 9, 055321. [CrossRef]

24. Polyakov, A.; Cabrini, S.; Dhuey, S.; Harteneck, B.; Schuck, P.; Padmore, H. Plasmonic light trapping in nanostructured metal surfaces. Appl. Phys. Lett. 2011, 98, 084801. [CrossRef]

25. Cao, A.; Zhang, K.; Zhang, J.; Liu, Y.; Kong, W. Actively tunable polarization-sensitive multiband absorber based on graphene. Chin. Phys. B 2020, 29, 114205. [CrossRef]

26. Johnson, P.; Christy, R. Optical Constants of the Noble Metals. Phys. Rev. B 1972, 6, 4370-4379. [CrossRef]

27. Lei, L.; Li, S.; Huang, H.; Tao, K.; Xu, P. Ultra-broadband absorber from visible to near-infrared using plasmonic metamaterial. Opt. Express 2018, 26, 5686-5693. [CrossRef] 
28. Yangchuk, B.; Zheludev, N.; Maier, S.; Halas, N.; Nordlander, P.; Giessen, H.; Chong, C. The Fano resonance in plasmonic nanostructures and metamaterials. Nat. Mater. 2010, 9, 707-715. [CrossRef]

29. Wu, F.; Liu, L.; Feng, L.; Xu, D.; Lu, N. Improving the sensing performance of double gold gratings by oblique incident light. Nanoscale 2015, 7, 13026-13032. [CrossRef]

30. Li, L.; Liang, Y.; Lu, M.; Peng, W. Fano Resonances in Thin Metallic Grating for Refractive Index Sensing with High Figure of Merit. Plasmonics 2015, 11, 139-149. [CrossRef]

31. Liu, H.; Zheng, L.; Ma, P.; Zhong, Y.; Liu, B.; Chen, X.; Liu, H. Metasurface generated polarization insensitive Fano resonance for high-performance refractive index sensing. Opt. Express 2019, 27, 13252-13262. [CrossRef] [PubMed]

32. Lin, G.; Yang, H.; Deng, Y.; Wu, D.; Zhou, X.; Wu, Y.; Cao, G.; Chen, J.; Sun, W.; Zhou, R. Ultra-compact high-sensitivity plasmonic sensor based on Fano resonance with symmetry breaking ring cavity. Opt. Express 2019, 27, 33359-33368. [CrossRef] [PubMed]

33. Zhang, Y.; Liu, W.; Li, Z.; Li, Z.; Cheng, H.; Chen, S.; Tian, J. High-quality-factor multiple Fano resonances for refractive index sensing. Opt. Lett. 2018, 43, 1842-1845. [CrossRef] [PubMed]

34. Wang, W.; Zheng, L.; Xiong, L.; Qi, L.; Li, B. High Q-factor multiple Fano resonances for high-sensitivity sensing in all-dielectric metamaterials. OSA Contin. 2019, 2, 2818-2825. [CrossRef]

Publisher's Note: MDPI stays neutral with regard to jurisdictional claims in published maps and institutional affiliations.

(C) 2020 by the authors. Licensee MDPI, Basel, Switzerland. This article is an open access article distributed under the terms and conditions of the Creative Commons Attribution (CC BY) license (http://creativecommons.org/licenses/by/4.0/). 